\title{
The Gaia-ESO Survey: an extremely Li-rich giant in globular cluster NGC 1261^
}

\author{
N. Sanna ${ }^{1}$, E. Franciosini ${ }^{1}$, E. Pancino ${ }^{1,2}$, A. Mucciarelli ${ }^{3,4}$, M. Tsantaki ${ }^{1}$, C. Charbonnel ${ }^{5,6}$, R. Smiljanic ${ }^{7}$, X. Fu ${ }^{8}$, \\ A. Bragaglia ${ }^{4}$, N. Lagarde ${ }^{9}$, G. Tautvaišiene ${ }^{10}$, L. Magrini ${ }^{1}$, S. Randich ${ }^{1}$, T. Bensby ${ }^{11}$, A. J. Korn ${ }^{12}$, A. Bayo ${ }^{13,14}$, \\ M. Bergemann ${ }^{15}$, G. Carraro ${ }^{16}$, and L. Morbidelli ${ }^{1}$
}

1 INAF - Osservatorio Astrofisico di Arcetri, Largo E. Fermi 5, 50125 Firenze, Italy e-mail: nicoletta.sanna@inaf.it

2 ASI Science Data Center, Via del Politecnico SNC, 00133 Rome, Italy

3 Dipartimento di Fisica e Astronomia, Università degli Studi di Bologna, Via Gobetti 93/2, 40129 Bologna, Italy

${ }^{4}$ INAF - Osservatorio di Astrofisica e Scienza dello Spazio di Bologna, via Gobetti 93/3, 40129 Bologna, Italy

5 Department of Astronomy, University of Geneva, Chemin des Maillettes 51, 1290 Versoix, Switzerland

${ }^{6}$ IRAP, UMR 5277, CNRS and Université de Toulouse, 14 Av. E. Belin, 31400 Toulouse, France

7 Nicolaus Copernicus Astronomical Center, Polish Academy of Sciences, ul. Bartycka 18, 00-716 Warsaw, Poland

8 The Kavli Institute for Astronomy and Astrophysics at Peking University, Beijing 100871, PR China

9 Institut UTINAM, CNRS UMR 6213, Univ. Bourgogne Franche-Comté, OSU THETA Franche-Comté-Bourgogne, Observatoire de Besançon, BP 1615, 25010 Besançon Cedex, France

10 Astronomical Observatory, Institute of Theoretical Physics and Astronomy, Vilnius University, Sauletekio av. 3, 10257 Vilnius, Lithuania

11 Lund Observatory, Department of Astronomy and Theoretical Physics, Box 43, 22100 Lund, Sweden

12 Observational Astrophysics, Division of Astronomy and Space Physics, Department of Physics and Astronomy, Uppsala University, Box 516, 75120 Uppsala, Sweden

13 Instituto de Física y Astronomía, Facultad de Ciencias, Universidad de Valparaíso, Av. Gran Bretaña 1111, 5030 Casilla, Valparaíso, Chile

14 Núcleo Milenio de Formación Planetaria - NPF, Universidad de Valparaíso, Av. Gran Bretaña 1111, Valparaíso, Chile

15 Max-Planck Institut für Astronomie, Königstuhl 17, 69117 Heidelberg, Germany

16 Dipartimento di Fisica e Astronomia, Università di Padova, Vicolo dell'Osservatorio 3, 35122 Padova, Italy

Received 18 May 2020 / Accepted 5 June 2020

\begin{abstract}
Lithium-rich stars in globular clusters are rare. Only 14 have been found so far, in different evolutionary phases from dwarfs to giants. Different mechanisms have been proposed to explain this enhancement, but it is still an open problem. Using spectra collected within the Gaia-ESO Survey, obtained with the GIRAFFE spectrograph at the ESO Very Large Telescope, we present the discovery of the first Li-rich star in the cluster NGC 1261, the second star known in the red giant branch bump phase. The star shows an extreme Li overabundance of $A(\mathrm{Li})_{\mathrm{LTE}}=3.92 \pm 0.14$, corresponding to $A(\mathrm{Li})_{\mathrm{NLTE}}=3.40 \mathrm{dex}$. We propose that the $\mathrm{Li}$ enhancement is caused by fresh Li production through an extra mixing process (sometimes referred to as cool bottom burning). Alternatively, it could be a preexisting Li overabundance caused by mass-transfer from a red giant star; this mechanism does not enhance the barium abundance and thus we observe low barium. To unambiguously explain the Li enhancement in globular cluster stars, however, a reliable determination of the abundance of key species like $\mathrm{Be},{ }^{6} \mathrm{Li},{ }^{12} \mathrm{C} /{ }^{13} \mathrm{C}$, and several $s$-process elements is required, as well as detailed modelling of chromospheric activity indicators.
\end{abstract}

Key words. surveys - techniques: spectroscopic - stars: chemically peculiar - globular clusters: individual: NGC 1261

\section{Introduction}

Lithium ( $\mathrm{Li})$ is a fragile element: it was synthesised during the Big Bang nucleosynthesis and is destroyed at relatively low temperatures $\left(\sim 2.5 \times 10^{6} \mathrm{~K}\right)$ in the stellar interiors (Reeves 1974). However, classical stellar evolution theory predicts that it is preserved in the stellar envelopes of metal-poor dwarf stars, with subsequent dilution mixing processes during the post-main sequence (MS) evolution. The investigation of $\mathrm{Li}$ abundances

\footnotetext{
^ Based on data obtained with the ESO VLT under the observing programmes 188.B-3002, 193.B-0936, and 197.B-1074.
}

in globular clusters (GCs; e.g. Mucciarelli et al. 2018, and reference therein) shows a complex situation: most dwarf stars have the same lithium abundance, $A(\mathrm{Li}) \simeq 2.2 \mathrm{dex}^{1}$, as found in most halo stars by Spite \& Spite (1982a,b), forming what is known as the Spite plateau. Mixing processes during the subgiant branch (SGB) cause a sharp drop in Li abundance by about 1 dex (see e.g. Fig. 3 in Mucciarelli et al. 2012). Then, during the red giant branch (RGB), stars between the first dredge-up and the RGB bump form another plateau at about $A(\mathrm{Li}) \simeq 1.0 \mathrm{dex}$

\footnotetext{
${ }^{1} A(\mathrm{Li})=\log [\mathrm{N}(\mathrm{Li}) / \mathrm{N}(\mathrm{H})]+12$.
} 

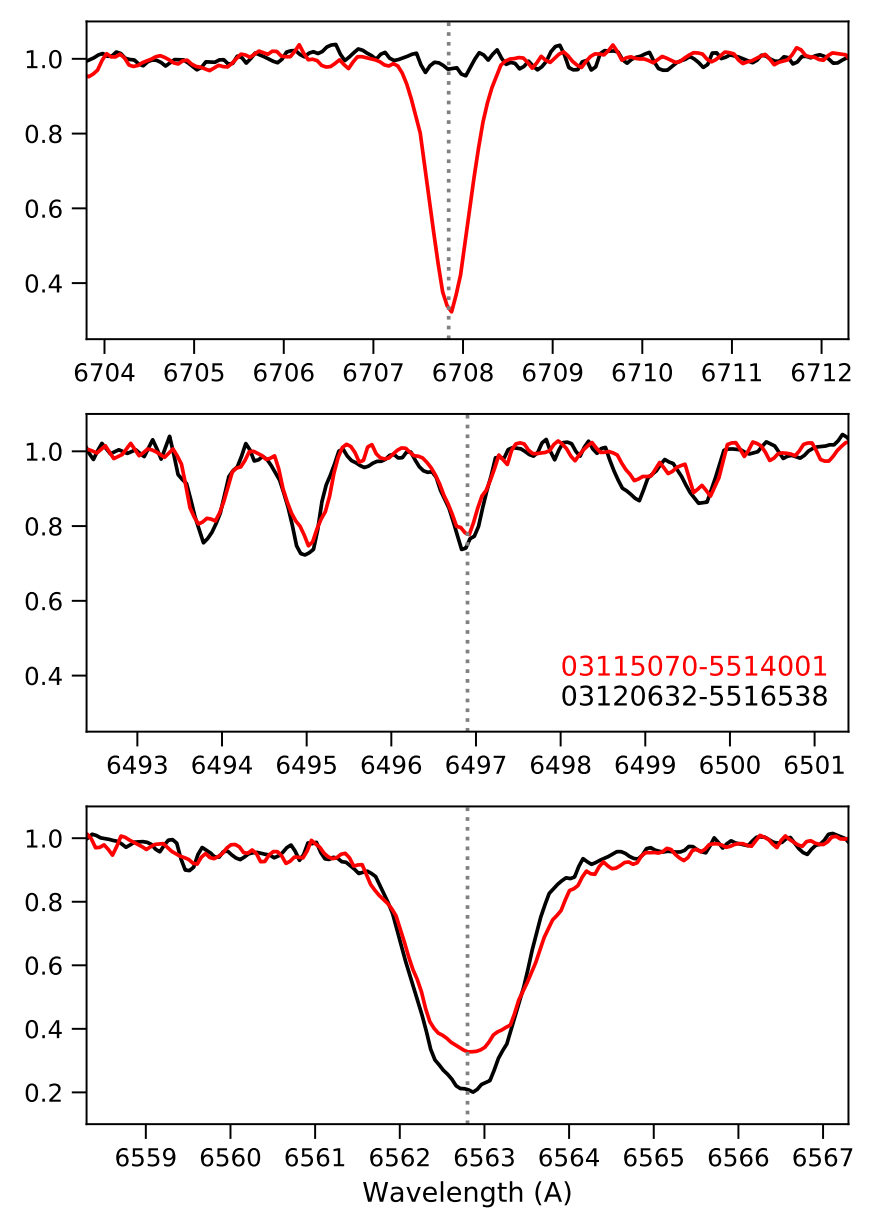

Fig. 1. Comparison of the spectrum of the Li-rich giant in NGC 1261 (red) with that of a normal giant with similar parameters (black) in the regions containing the Li doublet at $6707.8 \AA$ (top panel), the Ba II line at $6497 \AA$ (middle panel), and the $\mathrm{H}_{\alpha}$ line (bottom panel). The GES IDs of the two stars are indicated in the middle panel. See also Table 1.

(Gratton et al. 2000; Mucciarelli et al. 2012). After the bump, Li is diluted again (Charbonnel \& Zahn 2007). These results have been obtained analysing stars in different evolutionary phases, from the MS up to the asymptotic giant branch (AGB), in only a few GCs with different methods and number of stars. This observational behaviour is complicated in the case of GCs by the presence of multiple populations (Bastian \& Lardo 2018; Gratton et al. 2019). In some of the proposed scenarios the polluting material is almost $\mathrm{Li}$-free, and once diluted with $\mathrm{Li}$ normal gas should produce a lower Li abundance, but this is only found in some clusters (e.g. NGC 6752, Pasquini et al. 2005) and not in others, (e.g. NGC 362, D'Orazi et al. 2015a), where first and second population stars have the same $\mathrm{Li}$ abundance, or in $\omega$ Cen, where some second population stars have the same $\mathrm{Li}$ abundance as the first population and others do not (see Mucciarelli et al. 2018, and references therein, for details).

To this already quite complex framework, we have to add the existence of $14 \mathrm{Li}$-rich stars, i.e. that have a significantly increased Li abundance $(~ \gtrsim 0.5 \mathrm{dex})$ compared to normal GC stars in the same evolutionary phase. These stars are extremely rare (particularly in GCs, but also in open clusters and the field; see e.g. Casey et al. 2019) and for the moment they have also proved to be extremely difficult to explain. Four main possible enrichment paths have been explored and originally proposed to explain their high $\mathrm{Li}$ abundance:
- Engulfment of sub-stellar systems such as planets or brown dwarfs (Siess \& Livio 1999). This is valid especially for stars with metallicity higher than $\simeq-0.5 \mathrm{dex}$. Several studies (e.g. Gonzalez 1997; Fischer \& Valenti 2005; Casey et al. 2016) show that this scenario appears unlikely for metal-poor stars; other studies (e.g. Charbonnel \& Balachandran 2000) suggest that this scenario is unlikely because ${ }^{6} \mathrm{Li},{ }^{7} \mathrm{Li}$, and Be should be enhanced by the accretion at the same time, contrary to some observations (Balachandran et al. 2000). Moreover, the high Li overabundance $(A(\mathrm{Li}) \gtrsim 2.2$ dex $)$ observed in some stars is difficult to achieve by planet engulfment only (Aguilera-Gómez et al. 2016). Finally, most cases of Li-rich stars should be observed above the RGB bump, where the stars have larger radii and thus planet engulfment is more likely.

- Self-enrichment through the Cameron-Fowler mechanism (Cameron \& Fowler 1971). This could occur in two different evolutionary phases: (i) extra mixing during the RGB bump phase (sometimes called cool bottom processing; Boothroyd et al. 1995; Boothroyd \& Sackmann 1999; Palacios et al. 2001) when, thanks to the first dredge-up, the ${ }^{3} \mathrm{He}$-rich envelope is transported close to the hotter region of the H-burning shell and (ii) fresh $\mathrm{Li}$ production during the AGB, early-AGB, or AGB thermal pulses phases, depending on the mass of the star (Ventura \& D'Antona 2011; Charbonnel \& Balachandran 2000).

- Mass-transfer from different kinds of binary companions: (i) an AGB or super-AGB star, which also implies enrichment in the $s$-process elements (Busso et al. 1999; Ventura \& D'Antona 2011), depending on the AGB star mass or (ii) an RGB star that produces fresh Li thanks to the extra mixing process, but in this case no enrichment in $s$-process elements should be found.

- Ejecta during a nova outburst of a companion star. Numerical simulations predict that a high amount of ${ }^{7} \mathrm{Li}$ can be produced when a thermonuclear runaway occurs in the hydrogen envelope of the accreting white dwarf (Starrfield et al. 1978), and this possibility has been confirmed by the discovery of the first ${ }^{7} \mathrm{Li}$ detection in a nova (Izzo et al. 2015). A high enrichment of ${ }^{7} \mathrm{Be}$ is also expected during a nova outburst, as seems confirmed by the first detections (see e.g. Molaro et al. 2016).

In this framework, we present the discovery of a new Li-rich star in the GC NGC 1261 with Gaia-ESO Survey data. NGC 1261 is a GC in the Horologium constellation, with $[\mathrm{Fe} / \mathrm{H}]=-1.27$ dex and low extinction, $\mathrm{E}(\mathrm{B}-\mathrm{V})=0.01 \mathrm{mag}$ (Harris 2010). It is also one of the few GCs for which an extended stellar halo has been detected (Raso et al. 2020). This star is significantly more Li-rich than the bulk of the other Lirich stars discovered in GCs, similarly to the cases of NGC 6397 (Koch et al. 2011), NGC 4590 (Kraft et al. 1999, M68), and NGC 5272 (M3, Ruchti et al. 2011).

\section{Data analysis and results}

We based our work on the Gaia-ESO survey (hereafter GES; Gilmore et al. 2012; Randich \& Gilmore 2013) spectra. In particular, we used the FLAMES-GIRAFFE (Pasquini et al. 2000) spectra obtained with the HR15N (647-679 nm) set-up at medium resolution $(R=\lambda / \delta \lambda \simeq 20000)$, which includes the $\mathrm{Li}$ doublet at $6707.8 \AA$. The GES spectra reductions for GIRAFFE were performed with in-house software as described in detail by Jackson et al. (2015), resulting in one single spectrum for each star, combining different observations. The combined spectrum of the discovered Li-rich star in NGC 1261, with GES CNAME 03115070-5514001, is plotted in Fig. 1 along with a comparison star in the same cluster and with similar properties, 
Table 1. Estimates of relevant parameters for the Li-rich star in NGC 1261 and a comparison star (see Sect. 2 for details).

\begin{tabular}{lcc}
\hline \hline & Star & Star \\
CNAME & $03115070-5514001$ & $03120632-5516538$ \\
\hline RA (hh mm ss) & $03: 11: 50.70$ & $03: 12: 06.32$ \\
Dec $($ dd mm ss) & $-55: 14: 00.1$ & $-55: 16: 53.8$ \\
$S / N$ & 21 & 27 \\
$\mathrm{RV}\left(\mathrm{km} \mathrm{s}^{-1}\right)$ & $69.1 \pm 0.2$ & $69.7 \pm 0.2$ \\
$T_{\text {eff }}(\mathrm{K})$ & $4904 \pm 77$ & $4835 \pm 76$ \\
$\log g(\mathrm{dex})$ & $2.3 \pm 0.1$ & $2.2 \pm 0.1$ \\
$\left.v_{t}(\mathrm{~km} \mathrm{~s})^{-1}\right)$ & $1.5 \pm 0.2$ & $1.5 \pm 0.2$ \\
{$[\mathrm{Fe} / \mathrm{H}](\mathrm{dex})$} & -1.27 & -1.27 \\
$\mathrm{EW}(\mathrm{Li})(\mathrm{m} \AA)$ & $350 \pm 25$ & $20 \pm 5$ \\
$A(\mathrm{Li})_{\mathrm{LTE}}(\mathrm{dex})$ & $3.92 \pm 0.14$ & $0.86 \pm 0.10$ \\
$\Delta \mathrm{Li}$ NLTE $(\mathrm{dex})$ & -0.52 & +0.11 \\
$A(\mathrm{Ba})(\mathrm{dex})$ & $0.69 \pm 0.16$ & $0.96 \pm 0.17$ \\
\hline
\end{tabular}

03120632-5516538. The huge difference in the strength of the $\mathrm{Li}$ line at $6707.8 \AA$ is evident. The Li-rich star and the comparison star have a radial velocity of about 69.1 and $69.7 \mathrm{~km} \mathrm{~s}^{-1}$, respectively, fully compatible with the systemic velocity of NGC 1261 $\left(68.2 \pm 4.6 \mathrm{~km} \mathrm{~s}^{-1}\right.$, Harris 2010).

The two stars were observed in November 2017; therefore, they are not present in the publicly available GES DR3 release 2 (based on data observed until July 2014) nor in the current internal release, iDR5 (based on spectra acquired until December 2015). For the purpose of the present work, we have preliminarly estimated the relevant quantities (Table 1). The equivalent width (EW) of the Li $6707.8 \AA$ doublet was measured manually with IRAF $^{3}$. As can be seen, the star displays a Li absorption line that is more than 100 times stronger than that of a typical RGB star in NGC 1261. The Stetson et al. (2019) photometry was used to evaluate the star's position in the colour-magnitude diagram (CMD), showing that it lies precisely on the RGB bump (Fig. 2), using the bump estimates given by Ferraro et al. (1993) and Kravtsov et al. (2010). Using B-V and V-I colours from the Stetson et al. (2019) photometry and the colour-temperature calibrations by Alonso et al. (1999, 2001), we estimated the effective temperature $T_{\text {eff }}$ and the surface gravity $\log g$ (see Table 1).

We computed the $\mathrm{Li}$ abundance for the two stars using the spectral synthesis code SALVADOR developed by one of the authors (AM), based on the Kurucz abundance analysis routines (Kurucz 1993a,b; Sbordone et al. 2004) and using 1D ATLAS9 models, which assume local thermodynamical equilibrium (LTE). We assumed the Harris (2010) metallicity for NGC 1261 of $[\mathrm{Fe} / \mathrm{H}]=-1.27 \mathrm{dex}$ and a fixed micro-turbulent velocity of $v_{t}=1.5 \mathrm{~km} \mathrm{~s}^{-1}$, derived using the latest version of the Gaia-ESO calibration (Smiljanic et al. 2014, Worley et al., in prep.). We varied the ${ }^{6} \mathrm{Li}$ and ${ }^{7} \mathrm{Li}$ abundance in the region of the Li doublet at $6707.8 \AA$ to search for the best fit. For the comparison star, changing $v_{t}$ or the isotopic ratio implied variations of $\lesssim 0.01$ dex for the final Lithium abundance. For the Li-rich star, a $0.3 \mathrm{~km} \mathrm{~s}^{-1}$ variation in $v_{t}$ implied a variation of $0.03 \mathrm{dex}$ in the final $\mathrm{Li}$ abundance, and it was included in the

\footnotetext{
2 https://www.eso.org/qi/

3 IRAF is the Image Reduction and Analysis Facility, a general purpose software system for the reduction and analysis of scientific data IRAF is written and supported by the IRAF programming group at the National Optical Astronomy Observatories (NOAO) in Tucson, Arizona.
}

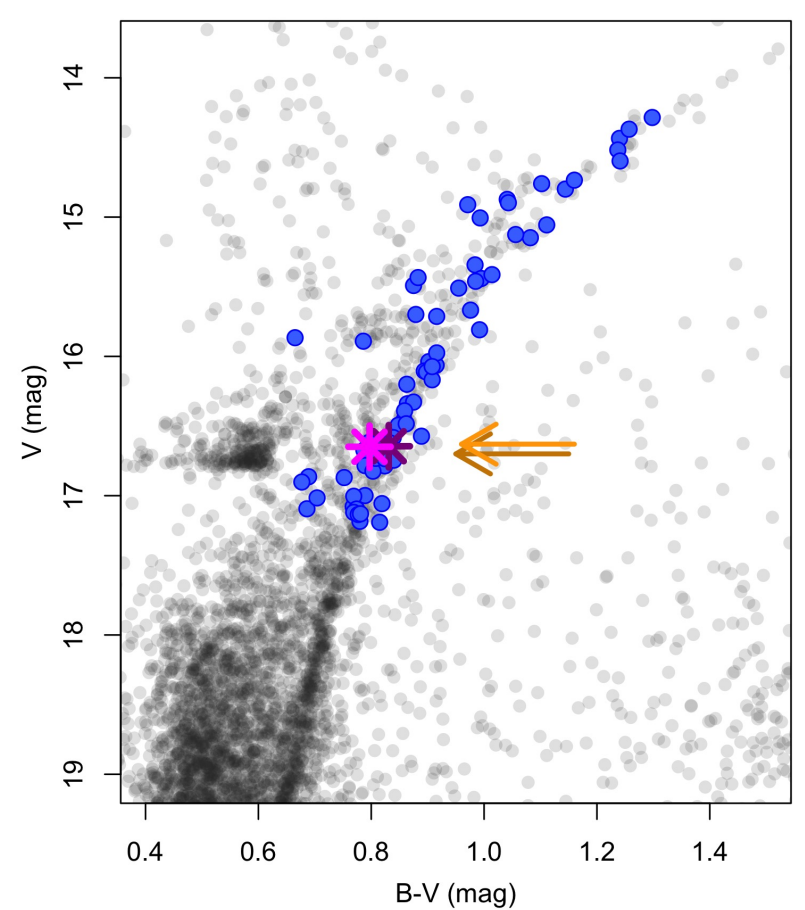

Fig. 2. Colour-magnitude diagram of NGC 1261 from Stetson et al. (2019, grey points) with the position of the Li-rich star (magenta star) and the comparison star (purple star). The other stars observed by GES with GIRAFFE are plotted in blue. The position of the RGB bump is also indicated, as determined by Ferraro et al. (1993, brown arrow) and Kravtsov et al. (2010, orange arrow).

final uncertainty computation and in the uncertainties on $T_{\text {eff }}$ and $\log g$. Most importantly, as observed by others, the choice of a solar ${ }^{6} \mathrm{Li} / \mathrm{Li}_{\text {tot }}$ value of 0.075 caused a poor fit of the asymmetric line profile and a final $\mathrm{Li}$ abundance 0.42 dex lower. In the case of Trumpler 5 , a ${ }^{6} \mathrm{Li} / \mathrm{Li}_{\text {tot }} \lesssim 2 \%$ was also found when using 3D modelling (Monaco et al. 2014). Assuming no ${ }^{6} \mathrm{Li}$, the fit of the line profile was significantly improved; in this case the final $\mathrm{Li}$ abundance is $A(\mathrm{Li})_{\mathrm{LTE}}=3.92 \pm 0.14 \mathrm{dex}$, almost 1000 times higher than the typical RGB star at the level of the bump. We also report in Table 1 the non-LTE correction to the lithium abundance for the two stars, obtained using the data by Lind et al. $(2009 a)^{4}$.

The HR15N set-up includes also the barium (Ba II) line at $6497 \AA$ and the $\mathrm{H}_{\alpha}$ Balmer line at $6563 \AA$ (middle and bottom panels of Fig. 1, respectively). Using the same spectral synthesis set-up used for $\mathrm{Li}$, we obtained $A(\mathrm{Ba})=0.69 \pm 0.16$ dex and $A(\mathrm{Ba})=0.96 \pm 0.17 \mathrm{dex}$ for the Li-rich star and the comparison star, respectively. As can be seen from the bottom panel of Fig. 1, the $\mathrm{H}_{\alpha}$ line of the Li-rich star is significantly shallower than the reference star and shows a marked asymmetry on the red wing that is quite different than in normal RGB stars. This anomalous profile is compatible with chromospheric activity and mass loss (Mészáros et al. 2009). Unfortunately, no asteroseismologic data are available for this star. It would be interesting to measure the projected rotational velocity as well, but with the GIRAFFE resolution we can only observe that there is no significant difference in the profile width of lines between the two stars. Unfortunately, there are no lines of elements (usually used to identify multiple populations in GCs) in the wavelength range covered by the HR15N set-up that was analysed. From the

\footnotetext{
4 http://inspect.coolstars19.com/
} 


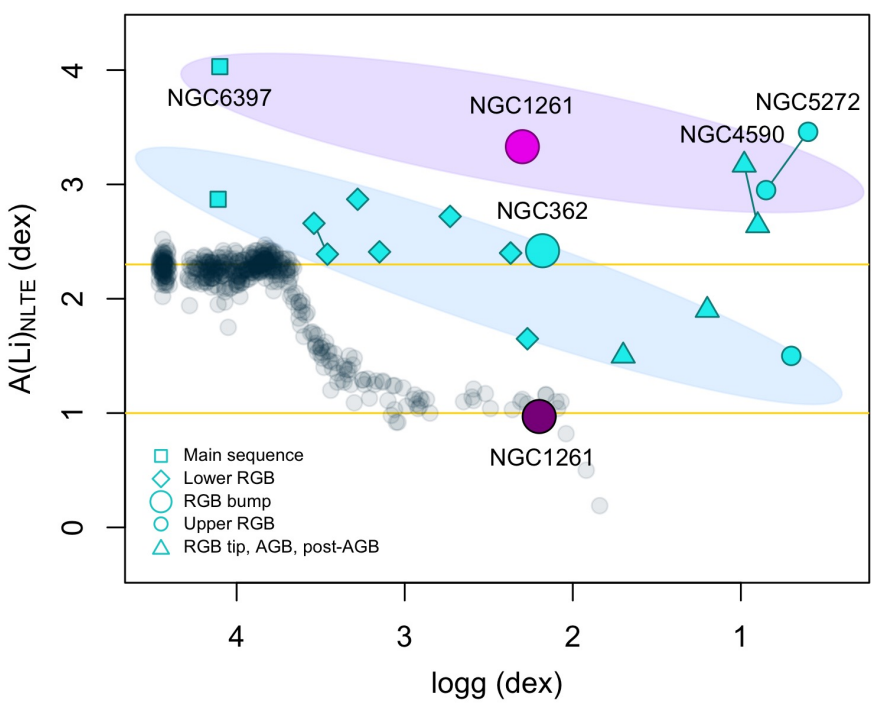

Fig. 3. Known Li-rich stars in GCs. Literature measurements are plotted as cyan symbols, our measurements for NGC 1261 are in magenta (for the Li-rich star) and purple (for the comparison star). Evolutionary phases are represented by different shapes as detailed in the legend. Different measurements of the same star are connected by segments. The Li-normal stars in NGC 6397 by Lind et al. (2009b) are shown in grey for comparison. The Spite and the low RGB plateaus are shown as yellow lines. Shaded regions represent the main group of Li-rich stars, enhanced by about $0.5-1.5$ dex compared to normal stars (cyan region) and the extremely Li-rich stars, about 1.5 dex above them (magenta region).

(U-B)-(B-I) photometric colour index the star seems to belong to the first population.

\section{Discussion and conclusions}

There are 14 Li-rich stars known in GCs, accounting for a small percentage of the stars studied spectroscopically: two dwarfs (Koch et al. 2011; Monaco et al. 2012), two AGB stars (Kirby et al. 2016); one post-AGB (Carney et al. 1998), and nine stars along different regions of the RGB or SGB (Kraft et al. 1999; Smith et al. 1999; Ruchti et al. 2011; D'Orazi et al. 2015b; Kirby et al. 2016; Gruyters et al. 2016; Mucciarelli et al. 2019). As can be seen from Fig. 3, most of the known Li-rich stars typically display an enhancement in the lithium abundance of 0.5-1.5 dex compared to normal stars in GCs, at least up to the RGB bump. Three of the literature stars, however, are significantly more Li-enhanced than the bulk of the sample and than the Spite plateau, by $\Delta A(\mathrm{Li}) \simeq 1-2 \mathrm{dex}$, as labelled in Fig. 3. One of these "super-Li-rich" stars is the dwarf star in NGC 6397, discovered by Koch et al. (2011), for which two possible origins have been proposed: mass transfer from an RGB companion or engulfment of a sub-stellar system. To date, the star in NGC 6397 is the only Li-rich star in GCs for which planet engulfment was considered (but see Pasquini et al. 2014). The other two are bright giants well above the RGB bump, in NGC 4590 (M 68; Ruchti et al. 2011; Kirby et al. 2016) and in NGC 5272 (M3; Kraft et al. 1999; Ruchti et al. 2011). Both the extra mixing process and mass-transfer from a companion have been proposed for these stars.

The Li-rich giant in NGC 1261 presented here is the fourth super-Li-rich star to be discovered. It clearly belongs to the RGB bump (Fig. 2), like the Li-rich star in NGC 362 (D'Orazi et al. 2015b), a GC with similar metallicity and horizontal-branch morphology, but the star in NGC 1261 displays a higher Li abundance: $A(\mathrm{Li})_{\mathrm{NLTE}}=3.40 \pm 0.14$ as opposed to $2.42 \pm 0.09$ dex. The Li abundance of the star in NGC 362 was explained as likely due to the extra mixing process, even if the authors also discuss the possibility that the star could belong to the pre-zero age horizontal branch, implying that it has already experienced the helium flash, because it lies slightly on the blue side or the RGB. This might apply also to our Li-rich star, but both stars appear closer to the RGB than to the horizontal branch. If we consider the extra mixing process, the different $A(\mathrm{Li})$ enrichment of the NGC 362 and NGC 1261 stars could be explained by observing that the NGC 362 star lies just above the RGB bump, where mixing mechanisms could have already started to occur, lowering $\mathrm{Li}$ and the ${ }^{12} \mathrm{C} /{ }^{13} \mathrm{C}$ ratio. If this is correct, then our Li-rich star in NGC 1261 should have a higher ${ }^{12} \mathrm{C} /{ }^{13} \mathrm{C}$ ratio, i.e. it should have reached the maximum $\mathrm{Li}$ enrichment just before mixing effects extend deep enough to lower the carbon ratio and to start destroying Li (Charbonnel \& Balachandran 2000).

To understand the origin of the Li-rich star in NGC 1261, we can count on three pieces of evidence: (1) the profile fit of the $\mathrm{Li}$ doublet suggests a very low ${ }^{6} \mathrm{Li} / \mathrm{Li}_{\text {tot }}$ value; (2) the Ba II abundance appears low and compatible with that of normal stars in NGC 1261; and (3) the $\mathrm{H}_{\alpha}$ line shows signs of chromospheric activity (central re-emission and asymmetric profile). The low Ba II abundance seems to suggest that we can exclude mass-transfer from an AGB companion, but we need more $s$ process elements to confirm this. Moreover, the absence of ${ }^{6} \mathrm{Li}$ in our best fit suggests that there has been fresh Li production. With the data in hand, our preferred hypothesis is that the $\mathrm{Li}$ enhancement of the RGB bump star in NGC 1261 is made by the extra mixing process because the star lies precisely on the RGB bump where this process is expected to occur. Unfortunately, this process does not explain the chromospheric activity suggested by the anomalous $\mathrm{H}_{\alpha}$ profile. The engulfment hypothesis of a sub-stellar body seems unlikely because it cannot explain the very high Li enrichment observed in the NGC 1261 star (Siess \& Livio 1999), but it would explain the chromospheric activity. Alternatively, mass transfer from an RGB companion (undergoing the extra mixing process) when the star was on the main sequence could also be a viable hypothesis. If this were the case, similarly to the AGB companion case, then the initial $\mathrm{Li}$ abundance of the star (before the first dredge-up) should have been of about $\simeq 4.6 \mathrm{dex}^{5}$, higher than the Li-rich star in NGC 6397. Moreover, as recently proposed, other kinds of interactions in binary systems could allow the Cameron-Fowler mechanism (Casey et al. 2019).

Additional information is clearly needed to unambiguously determine the origin of the $\mathrm{Li}$ enhancement in our NGC 1261 star and in the other 14 known Li-rich stars in GCs. In particular, accurate and precise $\mathrm{Be}$ and ${ }^{6} \mathrm{Li}$ abundances would allow us to evaluate the hypothesis of engulfment of a sub-stellar system. On the other hand, the ratio ${ }^{12} \mathrm{C} /{ }^{13} \mathrm{C}$ would be important to distinguish between production of fresh Li through extra mixing process and a pre-existing $\mathrm{Li}$ overabundance, perhaps resulting from binary mass transfer (Charbonnel \& Balachandran 2000). A full determination of $s$-process abundances could ultimately exclude the mass transfer hypothesis from an AGB companion, or help in pinpointing the mass range of the AGB donor (Busso et al. 1999). Additionally, measurement of the light elements that are known to anti-correlate in $\mathrm{GCs}(\mathrm{C}, \mathrm{N}, \mathrm{O}, \mathrm{Na}, \mathrm{Al}, \mathrm{Mg})$ would

\footnotetext{
$5 A(\mathrm{Li}) \simeq 1$ dex for a typical RGB bump star, while here $A(\mathrm{Li}) \simeq$ 3.4 dex. $3.4-1.0=2.4$. Adding this value to the Spite plateau $(2.2 \mathrm{dex})$, the star should have an initial abundance $\simeq 4.6$ dex.
} 
shed light on the relation between Li-rich stars and the presence of multiple stellar populations in GCs.

Acknowledgements. We thank the referee for the useful suggestions. NS and EP acknowledge the financial support to this research by INAF, through the Mainstream Grant 1.05.01.86.22 assigned to the project "Chemo-dynamics of globular clusters: the Gaia revolution” (P.I. E. Pancino). TB was partly funded by the grant 2018-04857 from the Swedish Research Council, and partly by the project grant "The New Milky Way" from the Knut and Alice Wallenberg Foundation. Based on data products from observations made with ESO Telescopes at the La Silla Paranal Observatory under programmes ID 188.B-3002, 193.B-0936, and 197.B-1074. These data products have been processed by the Cambridge Astronomy Survey Unit (CASU) at the Institute of Astronomy, University of Cambridge, and by the FLAMES/UVES reduction team at INAF/Osservatorio Astrofisico di Arcetri. These data have been obtained from the Gaia-ESO Survey Data Archive, prepared and hosted by the Wide Field Astronomy Unit, Institute for Astronomy, University of Edinburgh, which is funded by the UK Science and Technology Facilities Council. This work was partly supported by the European Union FP7 programme through ERC grant number 320360 and by the Leverhulme Trust through grant RPG-2012-541. We acknowledge the support from INAF and Ministero dell' Istruzione, dell' Università' e della Ricerca (MIUR) in the form of the grants "Premiale VLT 2012" and "Premiale 2015 MITIC". The results presented here benefit from discussions held during the Gaia-ESO workshops and conferences supported by the ESF (European Science Foundation) through the GREAT Research Network Programme.

\section{References}

Aguilera-Gómez, C., Chanamé, J., Pinsonneault, M. H., \& Carlberg, J. K. 2016, ApJ, 833, L24

Alonso, A., Arribas, S., \& Martínez-Roger, C. 1999, A\&AS, 140, 261

Alonso, A., Arribas, S., \& Martínez-Roger, C. 2001, A\&A, 376, 1039

Balachandran, S. C., Fekel, F. C., Henry, G. W., \& Uitenbroek, H. 2000, ApJ 542, 978

Bastian, N., \& Lardo, C. 2018, ARA\&A, 56, 83

Boothroyd, A. I., \& Sackmann, I. J. 1999, ApJ, 510, 232

Boothroyd, A. I., Sackmann, I. J., \& Wasserburg, G. J. 1995, ApJ, 442, L21

Busso, M., Gallino, R., \& Wasserburg, G. J. 1999, ARA\&A, 37, 239

Cameron, A. G. W., \& Fowler, W. A. 1971, ApJ, 164, 111

Carney, B. W., Fry, A. M., \& Gonzalez, G. 1998, AJ, 116, 2984

Casey, A. R., Ruchti, G., Masseron, T., et al. 2016, MNRAS, 461, 3336

Casey, A. R., Ho, A. Y. Q., Ness, M., et al. 2019, ApJ, 880, 125

Charbonnel, C., \& Balachandran, S. C. 2000, A\&A, 359, 563

Charbonnel, C., \& Zahn, J. P. 2007, A\&A, 476, L29

D’Orazi, V., Gratton, R. G., Angelou, G. C., et al. 2015a, MNRAS, 449, 4038

D'Orazi, V., Gratton, R. G., Angelou, G. C., et al. 2015b, ApJ, 801, L32

Ferraro, F. R., Clementini, G., Fusi Pecci, F., Vitiello, E., \& Buonanno, R. 1993, MNRAS, 264, 273
Fischer, D. A., \& Valenti, J. 2005, ApJ, 622, 1102

Gilmore, G., Randich, S., Asplund, M., et al. 2012, The Messenger, 147, 25 Gonzalez, G. 1997, MNRAS, 285, 403

Gratton, R. G., Sneden, C., Carretta, E., \& Bragaglia, A. 2000, A\&A, 354, 169

Gratton, R., Bragaglia, A., Carretta, E., et al. 2019, A\&ARv., 27, 8

Gruyters, P., Lind, K., Richard, O., et al. 2016, A\&A, 589, A61

Harris, W. E. 2010, ArXiv e-prints [arXiv:1012 . 3224]

Izzo, L., Della Valle, M., Mason, E., et al. 2015, ApJ, 808, L14

Jackson, R. J., Jeffries, R. D., Lewis, J., et al. 2015, A\&A, 580, A75

Kirby, E. N., Guhathakurta, P., Zhang, A. J., et al. 2016, ApJ, 819, 135

Koch, A., Lind, K., \& Rich, R. M. 2011, ApJ, 738, L29

Kraft, R. P., Peterson, R. C., Guhathakurta, P., et al. 1999, ApJ, 518, L53

Kravtsov, V., Alcaíno, G., Marconi, G., \& Alvarado, F. 2010, A\&A, 516, A23

Kurucz, R. 1993a, ATLAS9 Stellar Atmosphere Programs and $2 \mathrm{~km} / \mathrm{s}$ grid, Kurucz CD-ROM No. 13. Cambridge, 13

Kurucz, R. L. 1993b, SYNTHE spectrum synthesis programs and line data (Smithsonian Astrophysical Observatory)

Lind, K., Asplund, M., \& Barklem, P. S. 2009a, A\&A, 503, 541

Lind, K., Primas, F., Charbonnel, C., Grundahl, F., \& Asplund, M. 2009b, A\&A, 503,545

Mészáros, S., Avrett, E. H., \& Dupree, A. K. 2009, AJ, 138, 615

Molaro, P., Izzo, L., Mason, E., Bonifacio, P., \& Della Valle, M. 2016, MNRAS, 463, L117

Monaco, L., Villanova, S., Bonifacio, P., et al. 2012, A\&A, 539, A157

Monaco, L., Boffin, H. M. J., Bonifacio, P., et al. 2014, A\&A, 564, L6

Mucciarelli, A., Salaris, M., \& Bonifacio, P. 2012, MNRAS, 419, 2195

Mucciarelli, A., Salaris, M., Monaco, L., et al. 2018, A\&A, 618, A134

Mucciarelli, A., Monaco, L., Bonifacio, P., et al. 2019, A\&A, 623, A55

Palacios, A., Charbonnel, C., \& Forestini, M. 2001, A\&A, 375, L9

Pasquini, L., Avila, G., Allaert, E., et al. 2000, in FLAMES: a multi-object fiber facility for the VLT, SPIE Conf. Ser., 4008, 129

Pasquini, L., Bonifacio, P., Molaro, P., et al. 2005, A\&A, 441, 549

Pasquini, L., Koch, A., Smiljanic, R., Bonifacio, P., \& Modigliani, A. 2014, A\&A, 563, A3

Randich, S., Gilmore, G., \& Gaia-ESO Consortium 2013, The Messenger, 154, 47

Raso, S., Libralato, M., Bellini, A., et al. 2020, ApJ, 895, 15

Reeves, R. 1974, ARA\&A, 12, 437

Ruchti, G. R., Fulbright, J. P., Wyse, R. F. G., et al. 2011, ApJ, 743, 107

Sbordone, L., Bonifacio, P., Castelli, F., \& Kurucz, R. L. 2004, Mem. Soc. Astron. It., 5, 93

Siess, L., \& Livio, M. 1999, MNRAS, 308, 1133

Smiljanic, R., Korn, A. J., Bergemann, M., et al. 2014, A\&A, 570, A122

Smith, V. V., Shetrone, M. D., \& Keane, M. J. 1999, ApJ, 516, L73

Spite, F., \& Spite, M. 1982a, A\&A, 115, 357

Spite, M., \& Spite, F. 1982b, Nature, 297, 483

Starrfield, S., Truran, J. W., Sparks, W. M., \& Arnould, M. 1978, ApJ, 222, 600

Stetson, P. B., Pancino, E., Zocchi, A., Sanna, N., \& Monelli, M. 2019, MNRAS, 485,3042

Ventura, P., \& D'Antona, F. 2011, MNRAS, 410, 2760 